\title{
Coagulación intravascular diseminada como forma de presentación de leucemia aguda promielocítica: evaluación y tratamiento
}

\author{
Celia Martínez Vázquez 1,*, Marta Callejas Charavia 1, Patricia García Ramírez ${ }^{1}$, Julio García \\ Suarez ${ }^{1}$ \\ 1 Hematología y Hemoterapia, Hospital Universitario Príncipe de Asturias, Alcalá de Henares; Facultad de \\ Medicina y Ciencias de la Salud, Universidad de Alcalá. \\ * Autor correspondencia: celia.mv94@gmail.com
}

DOI: https://doi.org/10.37536/RIECS.2021.6.1.265

Recibido: 07/05/2021; Aceptado: 20/05/2021; Publicado: 31/05/2021

\begin{abstract}
Resumen: Presentamos el caso de un varón de 29 años ingresado en nuestro centro por hematuria macroscópica, coagulopatía y leucotrombopenia. Se estableció el diagnóstico inicial de coagulación intravascular diseminada (CID) aguda. El proceso diagnóstico para definir la causa de la CID confirmó la existencia de una Leucemia Promielocítica Aguda (LPA). La coagulopatía asociada a LPA es compleja, con datos biológicos de CID e hiperfibrinolisis primaria. Esta coagulopatía se asocia con hemorragias y se observa en el momento del diagnóstico, agravándose con la quimioterapia antileucémica. Constituye una emergencia médica, con una mortalidad cercana al $10 \%$ si no se maneja de forma adecuada. Actualmente, la LPA se trata con agentes no citotóxicos como el Ácido Transretinoico (ATRA) y el Trióxido de Arsénico (ATO), con una disminución de las tasas de sangrado. Por lo que un diagnóstico y tratamiento precoz de la coagulopatía suponen un aumento de la supervivencia en estos pacientes.
\end{abstract}

Palabras Clave: Hipofibrinogenemia, Coagulopatía intravascular diseminada, Leucemia Aguda Promielocitica, Ácido Transretinoico, Trióxido de Arsénico.

Abstract: We present the case of a 29-year-old man admitted to our center for hematuria with coagulopathy, mild leukopenia and moderate thrombopenia. A diagnosis of disseminated intravascular coagulation (DIC) secondary to Acute Promyelocytic Leukemia (APL) was made. Bleeding episodes due to DIC are one of the main causes of mortality at diagnosis in patients with APL. Currently there are lines of treatment with Arsenic Trioxide (ATO) and trans-retinoic acid (ATRA) that have replaced conventional chemotherapy regimens, with a decrease in bleeding rates. Therefore, an early diagnosis and treatment of DIC entails an increase in survival in these patients.

Key words: Hypofibrinogenemia, Disseminated intravascular coagulation, Acute Promyelocytic Leukemia, All-trans retinoic acid, Arsenic Trioxide. 


\section{Introducción y presentación del caso.}

Presentamos el caso de un varón de 29 años ingresado en nuestro centro por hematuria y coagulopatía con hipofibrinogenemia y elevación de dímero-D, así como leucotrombopenia moderada.

Se trata de un paciente fumador, sin otros antecedentes de interés, que acudió al servicio de urgencias por episodio de hematuria macroscópica de 3 días de evolución sin síndrome miccional asociado. Así mismo, presentaba pérdida de $6 \mathrm{~kg}$ de peso en el último mes. El cuadro se inició de forma espontánea sin relación con traumatismo o ejercicio. No refería antecedentes de diátesis hemorrágica previos.

Durante su valoración en Urgencias, la exploración física fue anodina con constantes vitales normales. No se palpaban adenopatías ni hepatoesplenomegalia. En las pruebas complementarias destacaba una hematuria macroscópica ( $>300$ hematíes/campo). Además, en el hemograma presentaba leucopenia leve (Leucocitos: $2.7 \times 10^{9} / \mathrm{L}$ con neutrófilos $1.65 \times 10^{9} / \mathrm{L}$ ), linfocitos $0.840 \times 10^{9} / \mathrm{L}$ y Monocitos 0.08 x109/L), trombopenia moderada (Plaquetas: 72 x109/L), y sin anemia ( $\mathrm{Hb}: 15.0 \mathrm{gr} / \mathrm{dl}$ ). En el frotis de sangre periférica se observaban abundantes equinocitos y aislados esquistocitos. No se observaban células inmaduras. En la bioquímica no se objetivaron alteraciones. El estudio de coagulación presentaba datos de coagulopatía, con Tiempo de Protrombina alargado (TP: 61 segundos), APTT normal, hipofibrinogenemia (65 mg/dl; valor normal: 200-400), Dímero-D muy elevado $(17.2 \mathrm{mg} / 1$; valor normal $<0,5)$. Se realizó PCR para SARS- CoV-2 así como serología viral para hepatitis, citomegalovirus, virus de Epstein-Barr y Leishmania que resultaron negativos. El paciente fue valorado por el servicio de Urología que descartó crisis-renoureteral como primera posibilidad, iniciándose sueroterapia con aclaramiento progresivo de orina.

Dados los hallazgos clínicos y biológicos, el paciente ingresó a cargo de Medicina Interna con juicio clínico inicial de CID. Se inició tratamiento con Concentrados de Fibrinógeno intravenoso para mantener unos niveles plasmáticos de Fibrinógeno por encima de $100 \mathrm{mg} / \mathrm{dl}$ y se realizó TAC toracoabdomino- pélvico, sin hallazgos de interés. Los hemocultivos fueron negativos. Durante el ingreso, se agravaron las citopenias por lo que se realizó estudio de médula ósea visualizándose una infiltración masiva $(82 \%)$ por células inmaduras y promielocitos atípicos muy positivos para la mieloperoxidasa (Figura 1) por lo que se informó de probable Leucemia Aguda Promielocitica (LPA, M3 según la FAB). Este diagnóstico se confirmó mediante biología molecular, con la detección del reordenamiento entre los genes PML y RAR $\alpha$ tipo BCR1.

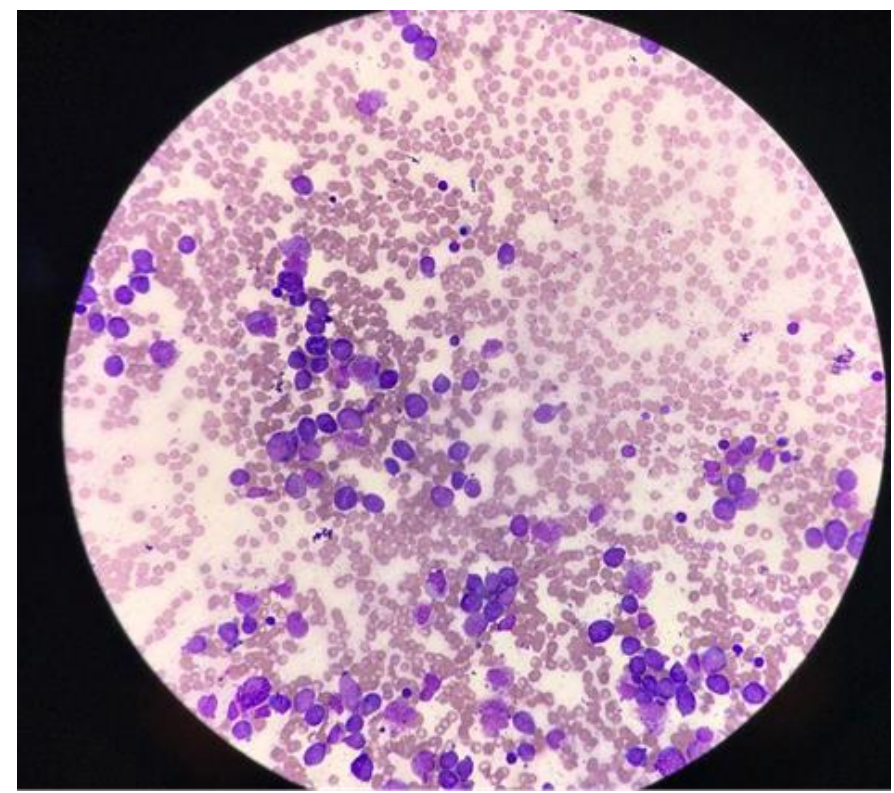

Figura 1 Imagen de aspirado de médula ósea donde se observa infiltración por blastos y promielocitos atípicos mieloperoxidasa positivos. 
Con el diagnóstico de LPA con t(15;17)(q22;q12); PML-RARA (clasificación OMS 2016) y CID asociada se trasladó al paciente al Servicio de Hematología y Hemoterapia y se inició tratamiento de inducción con Ácido Transretinoico (ATRA) y Trióxido de Arsénico (ATO) según protocolo PETHEMA LPA2017 para LPA de riesgo bajo/intermedio, así como medidas de soporte (concentrados de fibrinógeno, transfusión de plaquetas para mantener una cifra por encima de 30 x109/L, hidratación vigorosa, prevención de la nefropatía por ácido úrico con alopurinol y profilaxis antifúngica con equinocandinas). La hematuria se resolvió a las $48 \mathrm{~h}$ del ingreso y la coagulopatía al 5ํㅜa de iniciar el tratamiento con ATRA y ATO. El paciente alcanzó remisión completa morfológica de su LPA a los 39 días del inicio del tratamiento de inducción.

\section{Diagnóstico diferencial y discusión}

El paciente presentaba clínica de hematuria y datos de laboratorio compatibles con CID aguda: trombocitopenia, prolongación del tiempo de protrombina, disminución marcada de la concentración de fibrinógeno, incremento de Dímero $\mathrm{D}$ y esquistocitos en el frotis de sangre periférica. Dado que no existe ningún test específico para el diagnóstico de CID, en todos los casos deben evaluarse conjuntamente las pruebas de coagulación y el contexto clínico [1].

En primer lugar, es necesario realizar con urgencia el diagnóstico diferencial de la CID aguda [2,3]. Para ello debemos descartar la existencia de sepsis, neoplasias malignas (especialmente LPA), enfermedad hepática grave, y microangiopatía trombótica (MAT). Esta última incluye tres entidades: púrpura trombótica trombocitopénica (PTT), síndrome hemolítico urémico (SHU) mediado por la toxina Shiga de Escherichia coli y síndrome hemolítico urémico atípico mediado por complemento (SHU atípico) [4]. Es importante señalar que los tumores sólidos diseminados, especialmente adenocarcinomas secretores de mucina (p. Ej., páncreas, estómago y ovario), se pueden asociar a cuadros de CID crónica (hasta un 15\% de los casos) [5] y cursan con niveles de fibrinógeno normales o ligeramente elevados, así como dímero D elevado.

La presentación clínica (ausencia de fiebre y focalidad infecciosa, tensión arterial normal y ausencia de datos de laboratorio compatibles con infección grave) y los hemocultivos negativos descartaron la existencia de sepsis. Por otro lado, la enfermedad hepática grave suele presentarse con evidencia etiológica clara ( $\mathrm{p}$. Ej., Hepatitis aguda, cirrosis alcohólica) y pruebas de función hepática anormales, aunque las transaminasas pueden normalizarse si la función hepática está gravemente alterada. Algunos autores consideran que los niveles de factor VIII son útiles porque el factor VIII no es producido por los hepatocitos y, por lo tanto, a menudo está disminuido en la CID y elevado en la enfermedad hepática grave. El cuadro clínico del caso presentado no impresionaba de estar en relación con fallo hepático.

La presencia de esquistocitos en el frotis, junto con trombocitopenia y tendencia al sangrado son datos comunes a la CID y la MAT, pero la fisiopatología y el manejo de ambos cuadros son muy diferentes. La CID se desarrolla por la activación anormal (y a menudo masiva) de los procesos de coagulación y fibrinólisis dentro de la vasculatura, lo que lleva a una coagulación y fibrinólisis continuas, lo que da lugar a manifestaciones clínicas complejas de hemorragia y / o trombosis. Pueden contribuir una variedad de procoagulantes que inician el proceso, como el factor tisular (FT), productos bacterianos, micropartículas y ADN libre de células (cfDNA) y proteínas de unión al ADN de las trampas extracelulares de neutrófilos (NET). La alteración de la vía de señalización endotelial angiopoyetina-TIE2 también juega un papel central en la patogenia de la CID. Sin embargo, en la MAT el factor desencadenante es una activación plaquetaria por lesión endotelial ya sea por diminución de ADAMS13 (PTT), producción de toxina Shiga (SHU típico) o alteración del complemento en el SHU atípico [6] generando una anemia hemolítica microangiopática (esquistocitos), trombocitopenia, fallo renal, afectación del SNC y de otros órganos [7]. A diferencia de la CID, los pacientes con MAT tienen pruebas de coagulación normales porque los trombos microvasculares en estas afecciones son principalmente trombos ricos en plaquetas y pobres en fibrina, y no se asocian con coagulopatía por consumo (es decir, TP, aPTT, Fibrinógeno y dímero D normales. Además, a diferencia de la CID, los pacientes con MAT tienden a tener cambios 
microangiopáticos más llamativos en el frotis de sangre y otras anomalías de laboratorio específicas (p. Ej., nivel de actividad de ADAMTS13 $<10 \%$ en PTT; anomalías del complemento en SHU atípico).

El diagnóstico de CID se basa en los hallazgos de coagulopatía y/o fibrinólisis en el contexto clínico apropiado. En el caso que presentamos, los datos analíticos eran compatibles con el diagnóstico de CID aguda (ISTH score $>5$ ) según los criterios de la Sociedad Internacional de Trombosis y Hemostasia (ISTH) [8] (Tabla I). Sin embargo, la CID comprende un diagnóstico clínico y de laboratorio complejo que no puede determinarse exclusivamente a partir de datos aislados de laboratorio [9]. Por tanto, es esencial investigar la presencia de una condición clínica subyacente. La presencia de neutropenia y trombocitopenia progresivas debe hacernos pensar en la presencia de una enfermedad maligna. En este caso, se estableció el diagnóstico de LPA. La LPA es un subtipo de Leucemia Mieloide Aguda (LMA) caracterizada por la translocación del gen PML (cromosoma 15) con el gen RAR $\alpha$ (cromosoma 17), que genera una proteína de fusión PML-RAR $\alpha$, la cual ocasiona un bloqueo en la diferenciación mieloide y una acumulación de promielocitos leucémicos. Es una entidad nosológica particularmente agresiva por su evolución hiperaguda y una coagulopatía inicial potencialmente fatal. Constituye una neoplasia única, que con tratamientos dirigidos suele alcanzar la curación, en algunos casos incluso sin exposición a quimioterapia citotóxica. Dentro de la clasificación $\mathrm{FAB}$, corresponde a M3 y M3v (por variante microgranular), en la clasificación OMS de 2016, integra el subgrupo de "LMA con anormalidades genéticas recurrentes" y de riesgo bajo de recaída [10]. Tiene una incidencia casi constante con respecto a la edad, predominando en adultos jóvenes, a diferencia de las otras LMA. Clínicamente, representa una emergencia médica con alta mortalidad temprana por hemorragia (14\%), [11] por lo cual debe ser tratada inmediatamente ante la sospecha diagnóstica.

Tabla I Criterios diagnósticos para CID de la ISHT. Score CID $\geq 5$.

\begin{tabular}{|l|l|l|}
\hline Variables & Rango & Puntos \\
\hline Plaquetas $(\mathbf{\times} \mathbf{1 0} / \mathbf{L})$ & $<50$ & 2 \\
& $50-100$ & 1 \\
\hline & $>100$ & 0 \\
\hline Dímero D & Aumento Fuerte $>10$ veces del valor normal & 3 \\
\hline & Aumento moderado $\leq 10$ veces del valor normal & 2 \\
\hline & No aumento & 0 \\
\hline TP ratio (segundos) & $\geq 6$ & 2 \\
\hline & $3-6$ & 1 \\
\hline & $\leq 3$ & 0 \\
\hline Fibrinógeno $(\mathbf{m g} / \mathbf{d l})$ & $\leq 100$ & 1 \\
\hline & $>100$ & 0 \\
\hline
\end{tabular}

\section{Conclusiones}

La CID es un proceso sistémico con el potencial de causar tanto trombosis como hemorragia. Puede presentarse como una emergencia médica, potencialmente mortal o un proceso crónico, subclínico, según la causa desencadenante del cuadro. La correcta identificación de una CID y la condición subyacente responsable de la misma, son fundamentales para un manejo adecuado. La CID se observa en un número significativo de pacientes con cáncer, especialmente LPA y adenocarcinomas diseminados productores de mucina. En nuestro caso, el rápido diagnóstico de LPA y su tratamiento evitaron un desenlace fatal. 
Conflictos de Intereses: Los autores no declaran conflicto de intereses.

\section{Abreviaturas}

Las siguientes abreviaturas son usadas en este manuscrito:

CID: coagulación intravascular diseminada.

LPA: Leucemia Promielocítica Aguda.

ATRA: Ácido Transretinoico.

ATO: Trióxido de Arsénico.

$\mathrm{Hb}$ : Hemoglobina.

TP: Tiempo de Protombina.

APTT: Tiempo de tromboplastina parcial activado.

PCR: Reacción en cadena de la polimerasa.

TAC: Tomografía Axial Computerizada.

FAB: grupo cooperativo Franco-Americano-Británico:

DD: dímero D

ISTH: International Society on Thrombosis and Haemostasis.

\section{Referencias Bibliográficas}

1. Levi M, Scully M; How I treat disseminated intravascular coagulation. Blood 2018; 131 (8): 845854. doi: https://doi.org/10.1182/blood-2017-10-804096

2. Levi M, Sivapalaratnam S. Disseminated intravascular coagulation: an update on pathogenesis and diagnosis, Expert Review of Hematology 2018, 11:8, 663-672, DOI: 10.1080/17474086.2018.1500173

3. Iba, T., Watanabe, E., Umemura, Y. et al. Sepsis-associated disseminated intravascular coagulation and its differential diagnoses. J Intensive Care 2019; 7, 32. https://doi.org/10.1186/s40560-019-0387-z

4. Wada, H., Matsumoto, T., Suzuki, K. et al. Differences and similarities between disseminated intravascular coagulation and thrombotic microangiopathy. Thrombosis J 2018; 16, 14. https://doi.org/10.1186/s12959-018-0168-2

5. Colman RW, Rubin RN. Disseminated intravascular coagulation due to malignancy. Semin Oncol 1990;17:172-86.

6. Grisaru S, Xie J, Samuel S, Hartling L, Tarr PI, Schnadower D, Freedman SB; Alberta Provincial Pediatric Enteric Infection Team. Associations Between Hydration Status, Intravenous Fluid Administration, and Outcomes of Patients Infected With Shiga Toxin-Producing Escherichia coli: A Systematic Review and Meta-analysis. JAMA Pediatr. 2017 Jan 1;171(1):68-76. doi: 10.1001/jamapediatrics.2016.2952.

7. Wada H, Matsumoto T, Yamashita Y. Natural history of thrombotic thrombocytopenic Purpura and hemolytic uremic syndrome. Semin Thromb Hemost. 2014;40:866-73.

8. Di Nisio M, Thachil J, Squizzato A. Management of disseminated intravascular coagulation: a survey of the International Society on Thrombosis and Haemostasis. Thromb Res. 2015;136(2):239.

9. Wada H., Thachil J., Di Nisio M., Mathew P., Kurosawa S., Gando S. Guidance for diagnosis and treatment of disseminated intravascular coagulation from harmonization of the recommendations from three guidelines. J Thromb Haemost. 2013;11:761-767. https://doi.org/10.1111/jth. 51215.

10. Daniel A. Arber, Attilio Orazi, Robert Hasserjian, Jürgen Thiele, Michael J. Borowitz, Michelle M. Le Beau, Clara D. Bloomfield, Mario Cazzola, James W. Vardiman; The 2016 revision to the World Health Organization classification of myeloid neoplasms and acute leukemia. Blood 2016; 127 (20): 2391-2405. doi: https://doi.org/10.1182/blood-2016-03-643544

11. Rodeghiero F, Avvisati G, Castaman G, Barbui T, Mandelli F. Early deaths and anti-hemorrhagic treatments in acute promyelocytic leukemia. A GIMEMA retrospective study in 268 consecutive patients. Blood 1990;75:2112-2117. 
(). (1)

(C) 2021 por los autores; Esta obra está sujeta a la licencia de Reconocimiento 4.0

Internacional de Creative Commons. Para ver una copia de esta licencia, visite http://creativecommons.org/licenses/by-nc-nd/4.0/. 